\title{
Infrastructural requirements for the implementation of autonomous trucks in open- pit mines
}

\author{
Sanaa Benlaajili $^{1,2,3^{*}}$, Fouad Moutaouakkil ${ }^{2}$, and Ahmed Chebak ${ }^{4}$ \\ ${ }^{1}$ Research Foundation for Development and Innovation in Science and Engineering, Casablanca, \\ Morocco \\ ${ }^{2}$ Engineering Research Laboratory (LRI), National and High School of Electricity and Mechanic \\ (ENSEM) Hassan II University, Casablanca, Morocco \\ ${ }^{3}$ Innovation Lab for Operations (ILO), Mohammed VI Polytechnic University (UM6P), Morocco \\ ${ }^{4}$ Green Tech Institute (GTI), Mohammed VI Polytechnic University (UM6P), Morocco
}

\begin{abstract}
The field of study of autonomous trucks especially in open pit mines has been the subject of considerable research over the past three decades. In the last ten years in particular, interest in this area has improved considerably. The implementation of autonomous systems is an integral organizational and cultural change. This work is a guideline for the safe and effective implementation of autonomous trucks in mines. It provides an integral view of the different approaches to implementing autonomous mining trucks with respect to safety criteria, operating costs, etc. It also presents the criteria and measures to be considered when maintaining the haulage routes (vertical/horizontal/combined alignment) that are a vital component for the success of an automation project. Mine site designers and planners should ensure that the design and construction of traffic areas for these trucks are compatible with autonomy and minimize interactions with personnel and non-autonomous equipment. A proposed infrastructural requirement for the autonomous truck zone is presented in this work.
\end{abstract}

\section{Introduction}

The mining industry is increasingly adopting automation as a safety and productivity factor and as a critical factor in making future mining methods sustainable [1-3]. To prepare for its wider adoption, the International Organization for Standardization (ISO) has begun developing a global safety standard, "ISO/NP 17757, Earth-moving machinery - Safety of autonomous machine systems"[4], which will set the standard for the development and use of automation technology in mining.

This work provides the safety requirements for the autonomous or semi-autonomous trucks used in open-pit mining operations. It specifies the safety criteria for the autonomous trucks and its associated system and infrastructure, including hardware and software, and

*Corresponding author: sanaa.benlaajili-etu@etu.univh2c.ma 
provides guidance for safe use in their defined functional environments during the production operating cycle. Thus, the separation of the autonomous fleet from the manual fleet is an essential component for the safety of the mine operators. Therefore, a good management of the interactions and access authorizations to the autonomous truck area is important.

In autonomous mining haulage systems, the haulage road network is an essential and vital component of the production process. As such, the underperformance of a haulage road will have an immediate impact on mine safety, productivity and costs. The safety, productivity and longevity of equipment all depend on well-designed, constructed and maintained haulage roads. A haul road for autonomous trucks must be well designed to provide a specific level of performance and its routine maintenance should be managed accordingly [5].

The following sections are organized as follows: The first section provides an overview of approaches to introducing this new technology into the mine environment; the second section is used to present the requirements in the autonomous area to guarantee safe and secure circulation of autonomous trucks. The third section provide the criteria and measures to be considered when maintaining the haulage roads (vertical/horizontal/combined alignment). The last section will conclude this work.

\section{The implementation of autonomous trucks in open-pit mines}

The success of a mining equipment automation project depends on the right implementation strategies, commitment and vision of the organization. It is important to identify the key critical success factors for an autonomous haulage truck project [6].

The autonomous trucks will be successfully accepted in a mining company if the demonstration clearly shows how the criteria for selecting the autonomous truck are linked to performance measures for project phases, such as implementation, start-up and operation [6]. There are three approaches cited in [7] for integrating trucks into a mining operation:

- Slow Implementation: A less risky but more expensive approach where implementation is extended over a long period of time with multiple checkpoints. This approach allows the mine to integrate research and development efforts.

- Phased implementation: A medium-risk, medium-cost approach where implementation is done in several stages with two or three checkpoints. This approach often involves the implementation of a mix of mature and agile solutions.

- Rapid Implementation: A riskier but less costly approach where the new Autonomous trucks system completely replaces the old one. This approach often involves the implementation of mature commercial solutions.

The choice of the appropriate approach to implementing the Autonomous Trucks system in a mine depends on several factors. Figure 1 shows how low or high regulatory representation, risk tolerance, autonomous operational maturity, cost pressure, schedule pressure, and support from business stakeholders can be used to determine the appropriate approach.

In order to be able to decide on the appropriate approach for implementing autonomous trucks in an open pit mine, it is therefore necessary to assess if the infrastructure is adequate for this new technology, if the risks are controlled and if the stakeholders in this automation project are prepared for this change. 


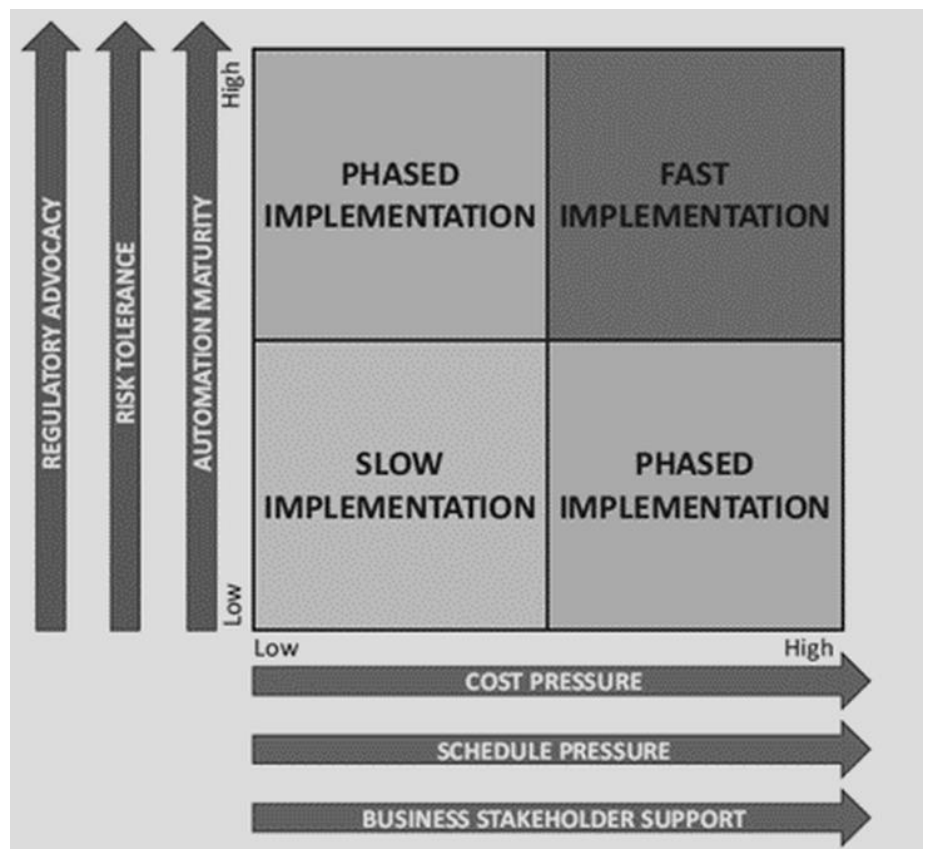

Fig. 1. Example of factors Influencing the Choice of an implementation approach [7]

\section{Autonomous Operating Zone (AOZ)}

This area for autonomous truck traffic should be well defined and delimited to prevent unauthorized access. Therefore, an authorization management system is essential.

The purpose of an access control system is to:

- Prevent people from entering the autonomous zone

- Prevent autonomous trucks from exiting the AOZ, whether in a controlled or uncontrolled manner.

Access control systems are connected to the central management system (fleet management system) to protect and prevent people or equipment from entering the area where the autonomous truck is operating.

\subsection{People's access to the Autonomous Operating Zone}

In order to be able to manage the access of people to the autonomous zone, a system of "RFID TAGs" must be assigned to each person operating in the mine. Once the truck detects the presence of a person in its environment, it must be maintained in a safe state, emits an audible alarm and alerts the control room. 


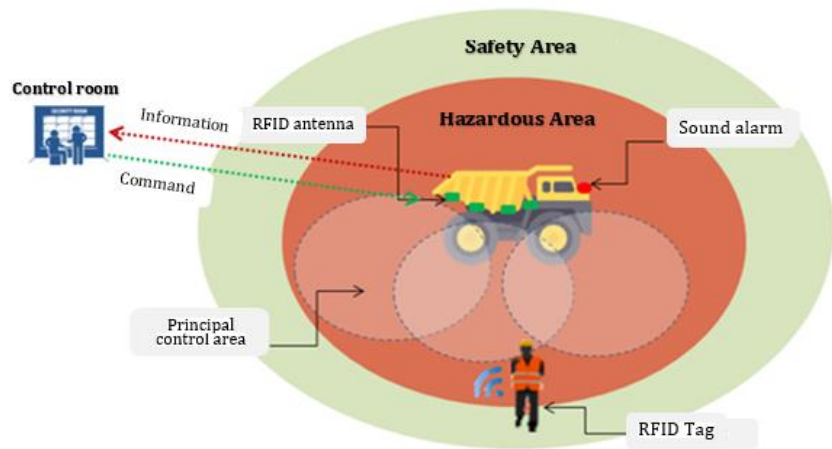

Fig. 2. Configuration scheme of the People's access control to the AOZ.

\subsection{Vehicle's access to the Autonomous Zone}

The risk assessment of the implementation of autonomous trucks in the haulage activity requires that each vehicle operating in the autonomous zone be monitored or escorted by a supervised person or vehicle. The following parameters should be considered [4]:

- The position of the vehicle;

- The speed of the vehicle;

- The minimum separation distance between the monitored vehicle and the autonomous truck;

- The maximum separation distance;

- The destination;

- The estimated duration in the autonomous zone.

This information is provided by equipping both light vehicles with GPS, network capabilities and emergency stops, which effectively interrupt the movement of the autonomous truck at the touch of a button. It is very important that all these systems are reliable to establish a safe and efficient operating environment. The Autonomous Truck Control System will effectively interrupt truck operations when other equipment enters the AOZ. Inaccurate locations can cause unwarranted interference in transportation operations. If the location of a heavy or light vehicle is completely lost (loss of GPS signal), the truck will automatically stop until the location can be re-established.

If a light vehicle moves in the vicinity of an autonomous truck, the system calculates if and when it will get into the path of the truck, slowing it down to avoid a collision.

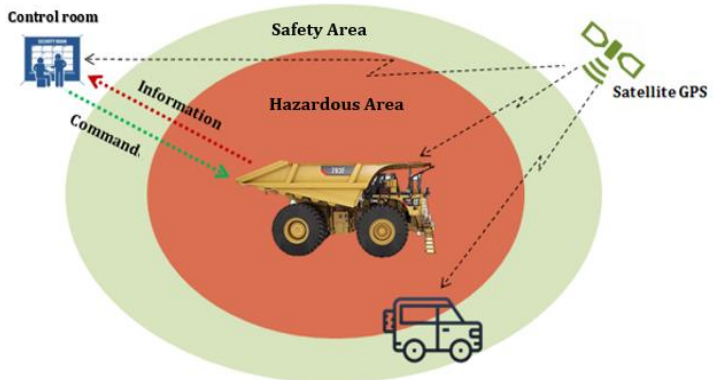

Fig. 3. Configuration scheme of the vehicle's access control to the AOZ 


\section{Design of haul roads for trucks in an open pit mine}

The design of an efficient haulage road for the movement of autonomous trucks in an open pit mine is of critical importance, as autonomous transportation systems require a well-developed infrastructure. All geometric elements of haul roads should be designed to allow safe and efficient travel at normal operating speeds. The ability of the autonomous vehicle to see ahead with all sensors for a distance equal to or greater than the required stopping distance is the overriding consideration. This section of the study discusses the effect of truck speed, grade and weight on stopping distance, as well as the design criteria for vertical and horizontal alignment [8].

\subsection{Vertical alignment}

Vertical alignment is the establishment of slopes and vertical curves that allow adequate stopping and viewing distances on all segments of the haulage road. It is not possible to create a safe autonomous transportation environment if slopes are designed without considering the braking limits of the autonomous truck in question $[8,9]$.

Braking distance

The manufacturer must confirm the distances required to stop the truck in accordance with ISO 3450:1996. ISO 3450:1996, which specifies braking system performance requirements and test procedures for earth-moving machinery and rubbertyred machines, is often used as a design standard by equipment manufacturers to allow uniform assessment of the braking capability of earth-moving machinery used in mines or on public roads. In the case of the autonomous truck, the braking performance must be measured from the moment the on-board control is received by the machine's brake subsystem until the machine stops [7].

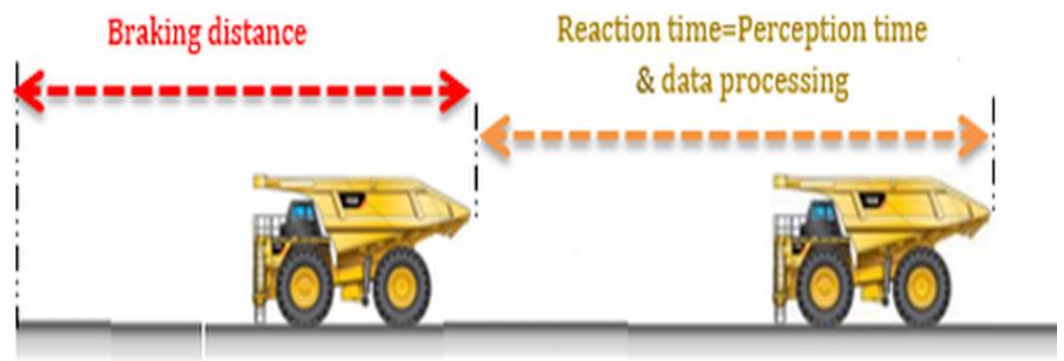

Fig. 4. Braking distance of autonomous truck

Due to the increased complexity, additional safety criteria are required [4]:

- The autonomous truck must have on board the ability to stop the machine;

- When the autonomous truck is operating in the AOZ, the control systems must be able to brake the machine while maintaining safe operation;

- The autonomous truck must have provisions to ensure that the safe operating temperatures and pressures of the braking and steering systems have been reached before the machine is put into autonomous mode.

\subsubsection{Sight distance and vertical curvature of the truck}

The sight distance of the autonomous truck can be defined as "the extent of the peripheral zone visible by all the perception sensors of the vehicle". It is therefore 
imperative that the sight distance be sufficient to allow the autonomous truck travelling at a given speed to stop before reaching a hazard. The distance measured between the perception sensors and the danger must always be equal to or greater than the required stopping distance.

The sight distance for the autonomous truck must be equal to or greater than the stopping distance of the vehicle.

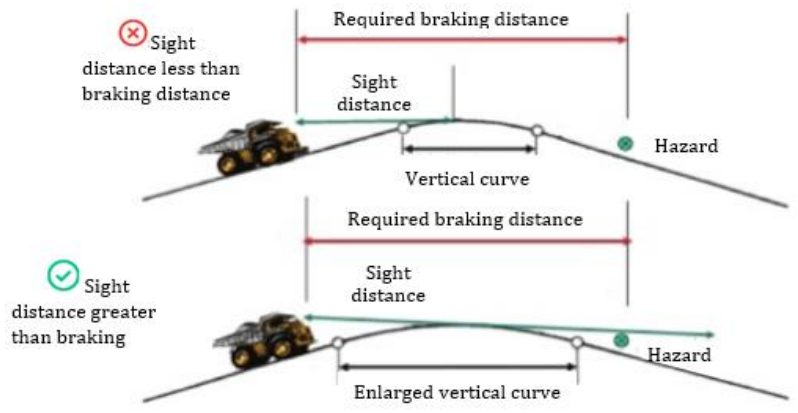

Fig. 5. Normal sight distance on a slope for autonomous driving

\subsubsection{Maximum safe slope limit}

From a safety point of view, the slopes of the haulage roads for autonomous trucks must be designed to adapt to the braking capacities of these machines with the lowest braking potential, due to their extreme weight and operating speed.

According to the Mining haul road design manual [8], slope reduction significantly increases the climbing speed of a mining haul truck. Thus, transport cycle times, fuel consumption, and stress on mechanical components, which lead to increased maintenance, can be reduced.

Slope has a major impact on the vertical line of sight requirements of the sensors. As the autonomous truck approaches the ramp, the coverage of obstacles and hazards in front of it is reduced. In addition, the slope geometry must be compensated for object detection; otherwise the ramps will be classified as obstacles and real obstacles outside the sensors' field of view will be missed.

The braking capabilities and perception performance of the autonomous truck must be evaluated and from this data the maximum slope on the road can be determined and must be extended for how many meters.

The speed of the autonomous truck at the beginning of the slope must be reduced and maintained throughout the descent.

Fig. 6. Scheme of the slope angle

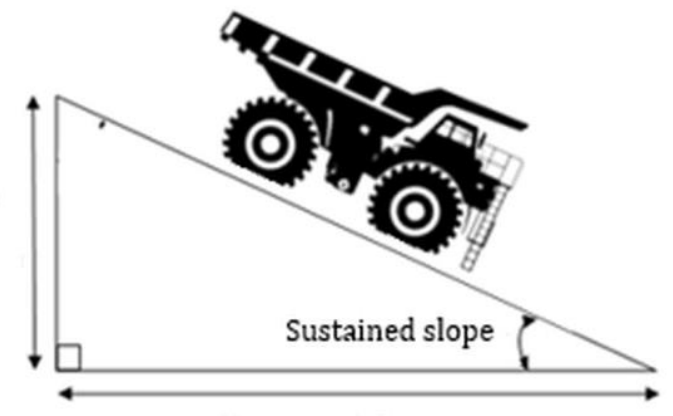

Horizontal distance 


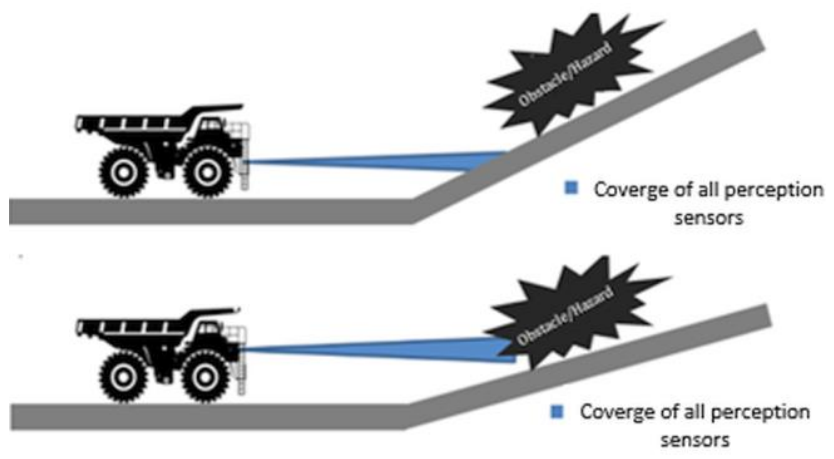

Fig. 7. Scheme of the impact of a slope on obstacle/hazard perception

\subsection{Horizontal alignment (Longitudinal)}

\subsubsection{Radius of curvature: Turning angle}

Horizontal curves must be designed in such a way that the autonomous truck will safely cross the curve at a given speed, taking into account the sight distance and the minimum turning radius [9]. The radius of curvature should be designed so that the centrifugal force exerted on the truck during rotation and the friction between the truck tire and the road surface are balanced.

In addition to the minimum radius of curvature, an elevation of the road grade is required to reduce the centrifugal forces exerted on the truck as it enters the curve.

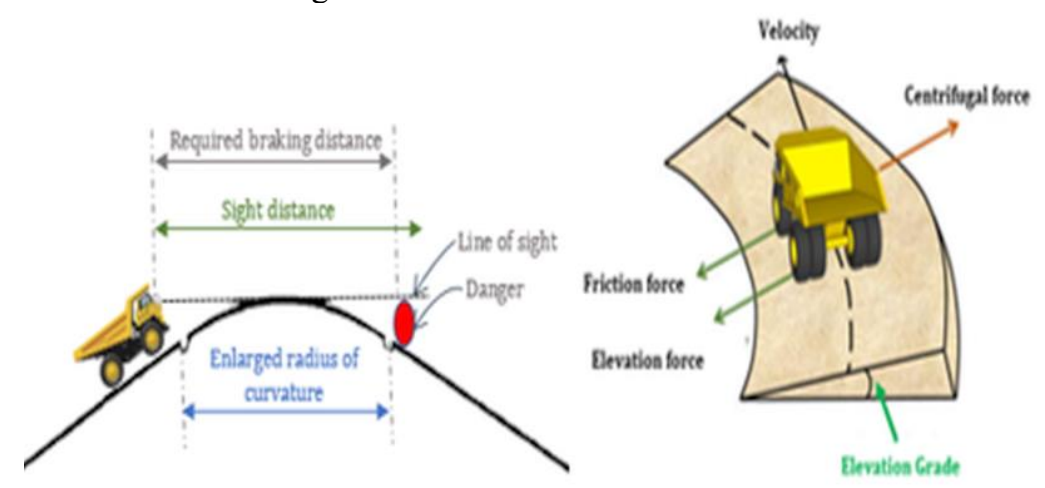

Fig. 8. Scheme of the radius of curvature Vs the elevation grade

\subsubsection{Road elevation and transverse slope of road}

When approaching a super-elevation turn from a straight section of road, there must be a gradual change in the level of the super-elevation of the road to allow the autonomous truck to move safely through the curve.

For a speed of the autonomous truck of $56 \mathrm{~km} / \mathrm{h}$, the recommended variation of the transverse slope is $5 \%$, therefore the total length of the curve should be $60 \mathrm{~m}$. One third of this length, $20 \mathrm{~m}$ should be adjusted at each end of the curve and the rest, $40 \mathrm{~m}$ applied to each tangent at the beginning and end of the curve. 


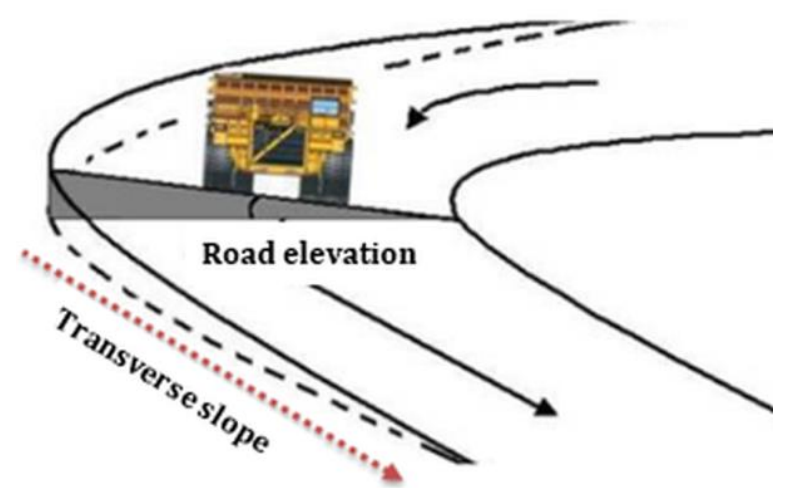

Fig. 9. Scheme of the road elevation and transverse slope of road

Width of roads

Roads that are too narrow can significantly reduce tire life by forcing the truck to drive over the berm when passing another vehicle. This results in damage to the sidewalls. When maintaining haul roads for autonomous trucks, sufficient leeway must be left at all times to promote safety and ensure continuity of the haul cycle. The width of the road must be sufficient for the number of lanes required. The largest trucks determine the width of the road.

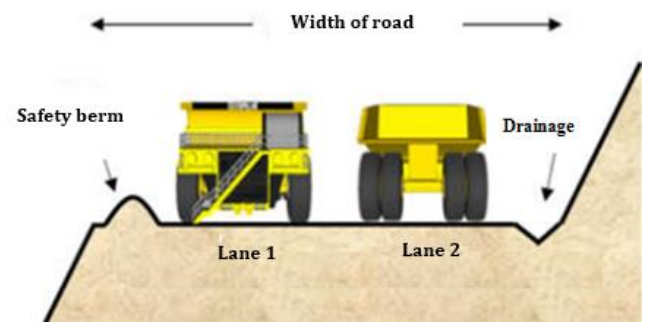

Fig. 10. Scheme of the width of road, drainage facility and safety berm

\subsubsection{Safety berms}

Safety berms are generally constructed from mine waste and are used to keep haulage trucks on track [10]. These berms will be more useful in the case of the stand-alone truck, it can always refer to the presence of these berms to keep it in its path.

The safety berm is generally constructed with intervals of 1 to 2 m wide spaced approximately $25 \mathrm{~m}$ apart to facilitate the drainage of the road surface. Drainage is excavated on each side of the road. 


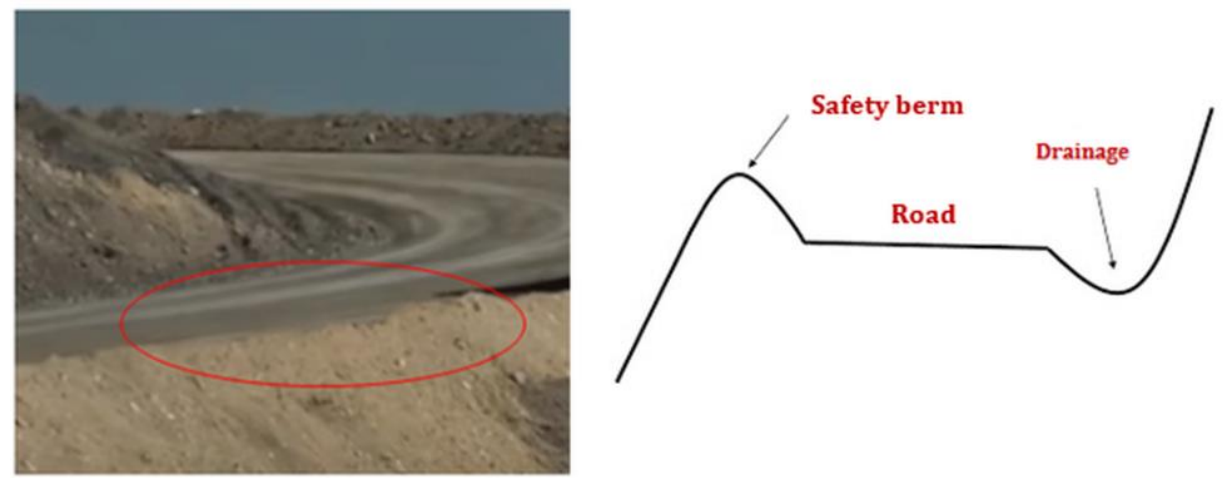

Fig. 11. Scheme of safety berms and drainage

\subsection{Combined alignment}

Poorly designed combinations of horizontal and vertical alignments can accentuate deficiencies and produce unexpected risks in the case of autonomous driving. If a horizontal curve is absolutely required, start it before the vertical curve [9].

Potential problem situations to be avoided are:

- Sharp horizontal turns at or near the top of a slope, as the sensors installed on the autonomous truck will have difficulty perceiving the angle of inclination of the slope.

- The autonomous truck should, at all times, be able to perceive the entire environment in front of it over a distance at least equal to the truck's braking distance

- Intersections should be as flat as possible.

- Horizontal curves should be raised by approximately $4 \%$ to $6 \%$ depending on the radius of curvature and the speed of the equipment.

- The radius of curvature must be greater than the minimum turning radius of the autonomous truck.

\section{Conclusion}

The mine operators focus more on the purely technical development of completely autonomous transport trucks and do not highlight the questions: how to implement these trucks in the mine's environment? What are the infrastructural requirements which are necessary to be satisfied to success the project of the introduction of autonomous mining trucks? Well designed and maintained haul roads are the key to minimizing autonomous truck haulage on-road hazards and costs, as well as increasing productivity. These systems also present new safety risks. For this reason, it is required to manage the area where the autonomous trucks will circulate and to manage the access authorizations to this area. In this paper, we have given answers to the previous questions, firstly outlining the approaches to introduce autonomous trucks in the haulage activity of a mine. Then we present the requirements for managing access authorizations to the autonomous zone. Finally, we presented the criteria and measures to be considered when maintaining the haulage roads (vertical/horizontal/combined alignment) that are a vital component for the success of an automation project. 
Work carried out within the framework of the Cooperation Agreement for technological and scientific development concluded between the Mohammed VI Polytechnic University (UM6P) and the Research Foundation for Development and Innovation in Science and Engineering (FRDISI). (CNRST).

This work was supported by the National Centre for Scientific and Technical Research

\section{References}

1. Un système de transport autonome optimise l'exploitation à ciel ouvert | Komatsu | Komatsu America Corp. https://www.komatsuamerica.com/autonomous-haulagesystem

2. Cat $\mid$ Realizing the Benefits of Autonomous Haulage | Caterpillar. https://www.cat.com/en_US/articles/customer-stories/mining/viewpoint/autonomoushaulage-gains.html

3. Cat $\mid$ Exploitation minière autonome : Sécurité et productivité $\mid$ Caterpillar. https://www.cat.com/fr_US/support/operations/technology/cat-minestar/minestar-inaction/auto-mining-safe-productivity.html

4. ISO 17757, 2017 : Engins de terrassement et exploitation minière -- Sécurité de système de machine autonome et semi-autonome

5. QNJAC Traffic Management in Quarries Guidance - 2013. https://www.safequarry.com/hotTopics/QNJAC\%20Traffic\%20Management $\% 20 \mathrm{in} \% 2$ 0Quarries\%20Guidance\%20-\%202013.pdf

6. J .Parreira, J .Meech Autonomous vs manual haulage trucks - how mine simulation contributes to future haulage system developments. $\mathbf{1 3}$

7. F .Harris Guideline for the implementation of autonomous systems in mining. Guideline for the implementation of autonomous systems in mining (2019)

8. WW . Kaufman, Ault JC Design of Surface Mine Haulage Roads - A Manual. 50

9. B. Regensburg, D. Tannant Guidelines for Mine Haul Road Design (2001)

10. J .Parreira An Interactive Simulation Model to Compare an Autonomous Haulage Truck System with a Manually-Operated System. 228 